\title{
Paul ERICKSON, Judy L. KLEIN, Lorraine DASTON, Rebecca LEMOV, Thomas STURM, and Michael D. GORDIN, How Reason Almost Lost Its Mind. The Strange Career of Cold War Rationality
}

Cléo Chassonnery-Zaïgouche

\section{(2) OpenEdition}

\section{Journals}

Édition électronique

URL : http://journals.openedition.org/ress/3561

DOI : $10.4000 /$ ress.3561

ISBN : 1663-4446

ISSN : 1663-4446

Éditeur

Librairie Droz

Édition imprimée

Date de publication : 30 novembre 2016

Pagination : 304-308

ISSN : 0048-8046

\section{Référence électronique}

Cléo Chassonnery-Zaïgouche, «Paul ERICKSON, Judy L. KLEIN, Lorraine DASTON, Rebecca LEMOV, Thomas STURM, and Michael D. GORDIN, How Reason Almost Lost Its Mind. The Strange Career of Cold War Rationality », Revue européenne des sciences sociales [En ligne], 54-2 | 2016, mis en ligne le 17 août 2016, consulté le 24 septembre 2020. URL : http://journals.openedition.org/ress/3561 ; DOI : https:// doi.org/10.4000/ress.3561

Ce document a été généré automatiquement le 24 septembre 2020.

(C) Librairie Droz 


\section{Paul ERICKSON, Judy L. KLEIN, Lorraine DASTON, Rebecca LEMOV, Thomas STURM, and Michael D. GORDIN, How Reason Almost Lost Its Mind. The Strange Career of Cold War Rationality}

Cléo Chassonnery-Zaïgouche

\section{RÉFÉRENCE}

Paul ERICKSON, Judy L. KLEIN, Lorraine DASTON, Rebecca LEMOV, Thomas STURM, and Michael D. GORDIN, 2013, How Reason Almost Lost Its Mind. The Strange Career of Cold War Rationality, Chicago, The University of Chicago Press, $272 \mathrm{p}$.

How Reason almost Lost Its Mind est un ouvrage extrêmement bien écrit par quatre historiens des sciences, une historienne de l'économie et un philosophe des sciences. Le livre retrace les contours du concept de rationalité dans le contexte des sciences sociales américaines des années de Guerre froide. Il ne s'agit pas tant de décrire l'émergence d'une théorie, d'une méthode ou d'une institution particulière que de faire l'analyse de «ce que signifie être rationnel durant l'ère de la possibilité de l'abîme nucléaire» (p. 21). Le livre se concentre sur ces hommes - tous impliqués dans des questions de défense, au Pentagone, dans les salles de séminaires de la RAND Corporation, derrière les vitres de la situation room de l'université de Harvard, ou encore sur le terrain des expériences nucléaires de Micronésie - et leurs conceptions de la rationalité : la confrontation de leurs idées et l'impact de ces débats sur le monde réel, ou, précisément, leur absence d'impact. 
2 Le trait le plus remarquable de l'ouvrage est d'insister sur le paradoxe entre l'extrême urgence à produire une science « utile » dans un contexte politique tendu et le type de méthodes employées - abstraites, universelles. La même idée préside à la présentation du contraste entre le contexte marqué d'incertitudes et la croyance en une définition strictement logique des processus sociaux. Le livre est ponctué d'histoire de pères de famille rationalisant les jeux d'enchères effectués avec leurs enfants, dont l'envergure théorique s'élargit ensuite, rappelant que « la mission [n'est] rien de moins que sauver le monde » (p. 24-25). Ces histoires récurrentes révèlent cette «inclinaison pour les analogies sauvages entre l'apocalyptique et le prosaïque » (p. 15) qui semble animer ces hommes d'action et définir leurs valeurs épistémiques.

Les deux premiers chapitres sont consacrés à l'émergence de la rationalité comme "ensemble complet de règles de comportement", pour reprendre la définition canonique de John Von Neumann et Oskar Morgenstern. Les quatre chapitres suivant proposent plusieurs articulations entre cette modélisation formelle et les débats en psychologie, en sociologie et en sciences politiques.

4 Pour analyser la manière dont la raison humaine a été reformulée autour de la notion de rationalité, le chapitre 1 revient sur trois éléments d'une histoire plus ancienne : les applications de la théorie des probabilités en dehors du champ de l'arithmétique au temps des Lumières, les tentatives de mécanisations du calcul au XIX ${ }^{e}$ siècle, et l'évolution du lien entre « règles » et raisonnement.

5 Malgré le développement de règles formelles de raisonnement, le concept de raison reste, au XVIII siècle, lié aux facultés de jugement et d'interprétation des individus. Les auteurs insistent sur l'opposition chez les premiers probabilistes entre le raisonnement et le fait de suivre mécaniquement des règles : les calculs, effectuées par des machines ou par des travailleurs non qualifiés - le plus souvent des femmes - apparaissent comme des soutiens au processus de raisonnement, non comme des substituts à celuici. Or, en sortant l'algorithme du domaine de la logique et en le transposant dans le domaine du choix et de la décision, le calcul sans intervention du jugement va précisément devenir le cœur de la notion de rationalité formelle du milieu du $\mathrm{xx}^{\mathrm{e}}$ siècle : "Whatever rationality was, it could be stated in algorithmic rules. » (p. 30).

6 Le chapitre 2 s'ouvre sur la narration pittoresque des tentatives de "programmation mathématique " pour "l'Opération Victuailles » (Operation Vittles) - le pont aérien de l'armée américaine visant à annuler les effets du blocus de Berlin. L'unité de contrôle statistique de l'armée de l'air américaine employait alors au sein du projet SCOOP (Project for the Scientific Computaion of Optimum Programs), des mathématiciens, comme George Dantzig, auteur d'un modèle de programmation linéaire, des économistes, des politistes, des «defense intellectuals » inclassables comme Robert McNamara, etc. Le but de l'équipe était de planifier les cadences optimales de ravitaillement afin de maximiser le tonnage par opération. Dues aux capacités de calcul très limitées - les calculatrices électromécaniques IBM disponibles en 1948 utilisent des cartes perforées sans possibilité d'y inscrire les larges matrices du modèle de Dantzig - l'opération fut planifiée avec des "protocoles sous-optimaux» (p. 53). In fine, le blocus de Berlin aura été plus utile pour le projet SCOOP que l'inverse, l'équipe se révélant incapable de fournir une planification des opérations au jour le jour. En revanche, les données empiriques reçues ont permis d'améliorer les modèles, les limitations de calcul générant nombre de métaphores pour la conceptualisation de la "rationalité limitée » de Herbert Simon (p. 70). L'épisode illustre parfaitement la constitution d'une «boîte à 
outils conceptuels» (p.79) appuyée sur une idée formelle de la rationalité qui caractérisera toute la période étudiée par l'ouvrage - des années 1950 aux années 1970. Les chapitres suivants sont consacrés à la confrontation entre cette conception de la rationalité et la "psychologie humaine ».

7 Le chapitre 3 est consacré aux analyses de la crise des missiles de Cuba de 1962. Contre les théories de la dissuasion nucléaire, Kennedy opte pour un blocus maritime ; s'en suit un processus de "désescalade » s'achevant par le retrait des missiles soviétiques. Le chapitre analyse deux études de cas : la stratégie de désarmement unilatéral de Charles E. Osgood - qu'il nomme GRIT pour "Graduated and Reciprocated Initiatives in Tension reduction» - et la conceptualisation des effets nocifs des dynamiques de groupe (ou groupthink) par Irving Janis. Ces deux approches psychologiques ont en commun de critiquer la théorie traditionnelle de "l'équilibre de la terreur " (ou Mutually Assured Destruction, MAD), dans la version qu'élabore Herman Kahn dans On Thermonuclear War (1960). Une dissuasion adéquate fournit le fondement objectif aux calculs de l'adversaire, qui doit s'abstenir de toute acte belligérant car toute attaque entrainerait alors des destructions irréversibles. Malgré la critique de la vision mécanique de la rationalité du MAD, les deux psychologues participent de cette reconfiguration de la rationalité autour de «règles » et de procédures. S'appuyant sur la psychologie de la perception, la GRIT est une technique qui consiste à unilatéralement décider et montrer des actes de conciliation. Pour Irving Janis, la "pensée de groupe » est une « détérioration de l'efficacité mentale [...] qui résulte de pressions de groupe » (p. 102). Le débarquement de la Baie des Cochons en est une illustration : cet événement est, selon Janis, le contre-exemple utilisé avec succès par Kennedy pour réorganiser le processus de délibération lors de la crise des missiles. Dans les deux cas, il s'agit d'élaborer des règles pour éviter que la psychologie n'affecte la «rationalité collective "; ces règles ne peuvent être définies qu'en réintroduisant des phénomènes psychologiques dans les débats sur la rationalité formelle.

8 L'objectif du chapitre 4 est de convaincre le lecteur que la notion de «situation » est centrale pour comprendre le type de rationalité formulée durant la Guerre froide. La situation, définie comme un mécanisme pour créer une cohérence dans un phénomène à observer, implique un certain nombre de dispositifs d'observation, d'appareils d'enregistrement, de protocoles expérimentaux, de séquences de formation et de volumineux manuels d'instruction. Des «bandes d'enfants de quatre ans » aux couples planifiant une fête d'anniversaire, des joueurs d'échecs aux professeurs organisant une soutenance de thèse, etc., autant de « situations » explorées pendant plus de 10 ans par Robert F. Bales derrière les vitres sans tain de la special room du Laboratoire des relations sociales de Harvard. Dans chacun de ces microcosmes où une décision doit être prise, Bales découvre une structure uniforme: chaque groupe devient un système de communication (p.125-129). Le chapitre s'ouvre cependant sur des expériences d'une autre échelle : le largage de 66 bombes nucléaires sur les atolls de Micronésie et les expériences intensives de collecte de données comportementales sur les îles alentour. Sur certains atolls, ces tests psychologiques et « moraux » ont été administrés quasiment à l'ensemble de la population locale (p. 108). Les 41 chercheurs en « sciences sociales et comportementales " avaient surtout pour objectif d'établir des standards afin de mesurer le plus rapidement possible des «profils psychologiques » (p. 109). Il n'est jamais fait référence aux essais nucléaires, comme si les "situations", laboratoires à ciel ouvert, ne partageaient pas la même géographie. Derrière cette conception de la rationalité repose la croyance que la salle de laboratoire ou une île du 
Pacifique occidental - pensées comme séparées du monde - forment de bonnes approximations du monde en général, "du moins pour des raisons opérationnelles » (p. 131).

9 Le chapitre 5 revient sur le destin étrange du dilemme du prisonnier, symbole de la Guerre froide, qui n'a pourtant pas immédiatement servi à analyser le «problème de la bombe». Les auteurs proposent d'expliquer l'attraction forte des sciences comportementales pour ce modèle par sa capacité à fournir des outils conceptuels extrêmement flexibles pour " coder » et penser les comportements humains dans une diversité de contextes; de la guerre du Vietnam à la chasse au cerf, de l'interpersonnel à l'international. Le chapitre est consacré à deux études de laboratoire des années 1960 - celle d'Anatol Rapoport sur la mesure de la coopération dans des jeux répétés et celle de Morton Deutsch sur l'autorité - et aux débats sur l'inclusion du dilemme du prisonnier en biologie évolutionnaire. L'agent rationnel typique qui émerge de l'ensemble de ces travaux semble avoir perdu les qualités associées à l'esprit et au raisonnement: «la mémoire, le choix, la conscience, l'orientation vers un but, l'intelligence » (p.157) et, dans certaines expériences, la capacité de communiquer.

Le dernier chapitre de l'ouvrage acte la fin de ce moment historique durant lequel la raison a presque perdu l'esprit en théorisant la rationalité formelle. Les auteurs prennent l'exemple de l'approche des "heuristiques et biais », souvent présentée comme un amendement empirique aux conceptions standards de la rationalité (notamment en économie). Cependant, alors que ces travaux ébranlent la valeur descriptive des théories formelles de la rationalité, ils semblent en accord avec sa « légitimité prescriptive» (p. 173). S'il évoque bien des continuités avec les versions précédentes de la rationalité, notamment concernant l'existence de règles universelles de comportement, ce chapitre raconte en réalité le début des "guerres de rationalité " des années 1980 et 1990 (p. 161). Les auteurs soulignent les aspects radicalement différentes du contexte - l'absence de consensus, la baisse des financements, la fin de la pluridisciplinarité du débat, etc. - la " profondeur, [l']ampleur, et [le] sens grisant et terrifiant de l'urgence » des débats ont en réalité disparu (p. 188).

D'autres histoires de cette conception de la rationalité auraient été possibles; ainsi, l'économie comme discipline est présente tout au long de l'ouvrage sans faire l'objet d'une étude de cas détaillée, ce qui a l'avantage d'offrir un regard différent sur certains objets, tels l'histoire de la théorie des jeux ou de l'économie comportementale. Le tableau de ce concept ambitieux est cependant extrêmement pénétrant.

\section{AUTEURS}

\section{CLÉO CHASSONNERY-ZAÏGOUCHE}

Université de Lausanne, Centre Walras-Pareto 